\title{
Pre-nova X-ray observations of V2491 Cygni (Nova Cyg 2008b)
}

\author{
A. Ibarra $^{1, \star}$, E. Kuulkers ${ }^{1}$, J. P. Osborne ${ }^{2}$, K. Page ${ }^{2}$, J. U. Ness ${ }^{1}$, R. D. Saxton ${ }^{1}$, W. Baumgartner ${ }^{3,4}$, V. Beckmann ${ }^{5,6}$,

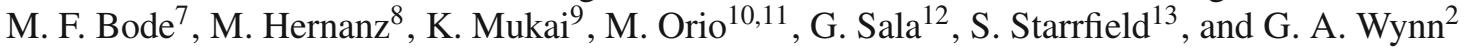 \\ 1 ESA, European Space Astronomy Centre (ESAC), Apartado 78, 28691 Villanueva de la Cañada (Madrid), Spain \\ e-mail: Aitor.Ibarra@sciops.esa.int \\ 2 The Department of Physics and Astronomy, The University of Leicester, Leicester, LE1 7RH, UK \\ 3 NASA/Goddard Space Flight Center, Code 662, Greenbelt, MD 20771, USA \\ 4 University of Maryland, Baltimore County, 1000 Hilltop Circle, Baltimore, MD 21250, USA \\ 5 ISDC Data Centre for Astrophysics, Chemin d'Écogia 16, 1290 Versoix, Switzerland \\ 6 Observatoire Astronimique de l'Université de Genève, Chemin des Maillettes 51, 1290 Sauverny, Switzerland \\ 7 Astroph. Research Institute, Liverpool John Moores Univ., Twelve Quays House, Egerton Wharf, Birkenhead CH41 1LD 7, UK \\ 8 IEEC-CSIC, Campus UAB, Facultat Ciències, C5-par, 2on, 08193 Bellaterra, Spain \\ 9 CRESST and X-ray Astrophysics Laboratory NASA/GSFC, Greenbelt, MD 20771, USA \\ 10 INAF - Osservatorio Astronomico di Padova, vicolo Osservatorio 5, 35122 Padova, Italy \\ 11 Department of Astronomy, University of Wisconsin, 475 N. Charter Str., Madison WI 53706, USA \\ 12 Grup d'Astronomia i Astrofísica, Dept. Física i Enginyeria Nuclear, Univ. Politècnica de Catalunya, 08036 Barcelona, Spain \\ 13 School of Earth \& Space Exploration, Arizona State University, PO Box 871404, AZ 85287-1404, USA
}

Received 23 January 2009 / Accepted 23 February 2009

\section{ABSTRACT}

\begin{abstract}
Classical novae are phenomena caused by explosive hydrogen burning onto an accreting white dwarf. Only one classical nova had been identified in X-rays before the actual optical outburst occurred (V2487 Oph). The recently discovered nova, V2491 Cyg, is one of the fastest $(\mathrm{He} / \mathrm{N})$ novae observed to date. Using archival ROSAT, XMM-Newton, and Swift data, we show that V2491 Cyg was a persistent X-ray source during its quiescent time before the optical outburst. We present the X-ray spectral characteristics and derive $\mathrm{X}$-ray fluxes. The pre-outburst X-ray emission is variable, and, at least in one observation, it exhibits a soft X-ray source.
\end{abstract}

Key words. X-rays: binaries - novae, cataclysmic variables

\section{Introduction}

Classical (and recurrent) novae are the third most energetic type of outburst after GRB and SNe, but are far more common than both of these catastrophic explosions. They take place in binary systems in which mass is transferred from a low-mass secondary star onto a white dwarf. The accreted material gradually becomes degenerate, and when temperatures become high enough and the pressure at the bottom of the accreted envelope is sufficient, a thermonuclear runaway results. Enough energy is deposited into the accreted material to eject a fraction of the envelope of the white dwarf (Bode \& Evans 2008).

Novae can be classified into two observational groups of Fe II and $\mathrm{He} / \mathrm{N}$, depending on the emission lines detected in their optical spectra (Williams 1992). Novae with prominent Fe II lines evolve more slowly, have lower levels of ionization, and exhibit P Cygni absorption components. Novae with stronger lines of $\mathrm{He}$ and $\mathrm{N}$ have larger expansion velocities and a higher level of ionization, and the lines are more flat-topped with little absorption. Only a few X-ray observations of $\mathrm{He} / \mathrm{N}$ novae exist and because of their rapid evolution little is known about them.

The nova V2491 Cyg (Nova Cyg 2008b) was discovered on $t_{0}=2008$ April 10.8 UT at about $7.7 \mathrm{mag}$ on unfiltered CCD frames (Nakano et al. 2008). Tomov et al. (2008a) found an optical $V / R / I$ decline rate from $\sim 0.3$ to 0.15 mag per day between $t_{0}+2.3$ and $t_{0}+7.3$ days. Assuming $t_{0}+0.6$ and $V=7.54$ mag for the maximum (Nakano et al. 2008), the time $t_{2}$

* INSA (Ingeniería y Sistemas Aeroespaciales S.A.). in the $V$ band is 4.6 days, where $t_{2}$ is defined as the elapsed time to decrease $2 \mathrm{mag}$ in its visual luminosity. This $t_{2}$ value makes V2491 Cyg a very fast nova, similar to V838 Her (O'Brien et al. 1994) and V2487 Oph (Hernanz \& Sala 2002). Optical postoutburst spectra showed V2491 Cyg to be a nova in its early phase of outburst (Ayani \& Matsumoto 2008). V2491 Cyg was classified as a He/N nova based on the photometric and spectroscopic results (Lynch et al. 2008; Tomov et al. 2008b; Helton et al. 2008). The fast decline and optical spectral characteristics, such as extremely broad lines with complex profiles, and large expansion velocities ( 4000-6000 $\mathrm{km} \mathrm{s}^{-1}$ ), imply V2491 Cyg that is a peculiar and extremely fast nova (Ashok et al. 2008; Lynch et al. 2008; Tomov et al. 2008a,b).

From optical measurements, the reddening towards the source was determined $E(B-V)=0.3$ (Lynch et al. 2008) and $E(B-V)=0.43$ (Rudy et al. 2008) on $t_{0}+2$ and $t_{0}+7$ days, respectively. Taking the latter value, using the correlation between visual extinction, $A_{V}$, and the dust (and hydrogen) column densities, assuming there is no intrinsic absorption $\left(N_{\mathrm{H}}\left[\mathrm{cm}^{2}\right] / A_{V}=\right.$ $1.79 \times 10^{21}$, e.g., Predehl \& Schmitt 1995), and using $A_{V}=$ $3.1 E(B-V)$, the optical reddening corresponds to $N_{\text {dust } / \mathrm{H}} \simeq 2.4 \times$ $10^{21} \mathrm{~cm}^{-2}$. Using the reddening of $E(B-V)=0.43$, a distance of $10.5 \mathrm{kpc}$ was estimated by Helton et al. (2008).

The object was fainter than 14 mag at $t_{0}-2$ days (Nakano et al. 2008). Between five and nine months prior the outburst, Balman et al. (2008) did not detect the pre-nova to $R=18.6 \pm$ 0.5 . However, the pre-nova was detected in archival plates spanning $\sim 16$ years showing a persistent source at $R \simeq 16.3$ 
Table 1. Pre-nova X-ray counterpart source identifications, coordinates, uncertainty, and offset $(\delta)$ from the position of the optical nova (19h43m01.96s, +32d19'13.8"; Nakano et al. 2008).

\begin{tabular}{cccc}
\hline \hline Name & $\begin{array}{c}\text { Position } \\
(\text { RA, Dec; J2000.0) }\end{array}$ & $\begin{array}{c}\text { Error } \\
(90 \%)\end{array}$ & $\delta$ \\
\hline 1RXS J194259.9+321940 & $19 \mathrm{~h} 42 \mathrm{~m} 59.9 \mathrm{~s},+32 \mathrm{~d} 19^{\prime} 40.5^{\prime \prime}$ & $28^{\prime \prime}$ & $37^{\prime \prime}$ \\
2RXP J194302.0+321912 & $19 \mathrm{~h} 43 \mathrm{~m} 02.0 \mathrm{~s},+32 \mathrm{~d} 19^{\prime} 12.0^{\prime \prime}$ & $4^{\prime \prime}$ & $1.9^{\prime \prime}$ \\
XMMSL1 J194301.9+321911 & $19 \mathrm{~h} 43 \mathrm{~m} 02.0 \mathrm{~s},+32 \mathrm{~d} 19^{\prime} 10.5^{\prime \prime}$ & $12^{\prime \prime}$ & $3.7^{\prime \prime}$ \\
Swift J194302.1+321913 & $19 \mathrm{~h} 43 \mathrm{~m} 02.0 \mathrm{~s},+32 \mathrm{~d} 19^{\prime} 11.0^{\prime \prime}$ & $3.7^{\prime \prime}$ & $2.8^{\prime \prime}$ \\
\hline
\end{tabular}

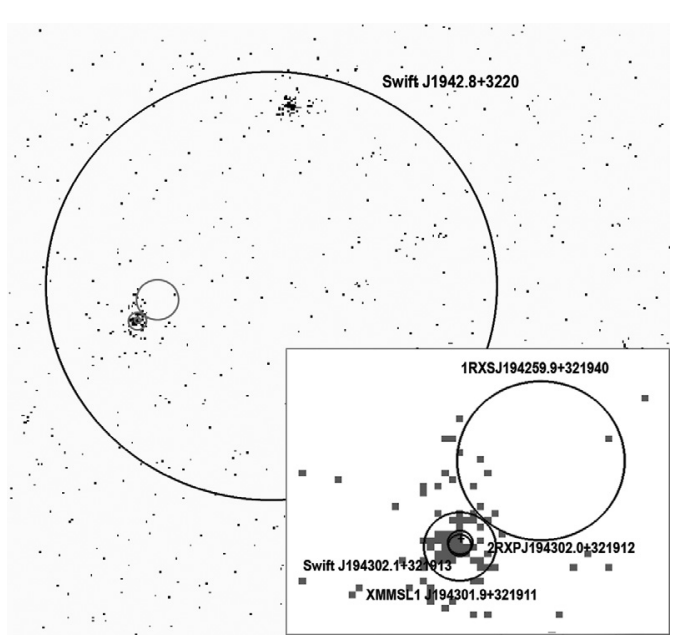

Fig. 1. Swift/XRT X-ray image of the V2491 Cyg field of view. The big circle corresponds to the Swift/BAT uncertainty and the zoomed view corresponds to the pre-nova X-ray counterpart uncertainties of Table 1. The cross corresponds to the optical coordinates.

(Jurdana-Sepic \& Munari 2008). This indicates a dimming of the source by about 2 mag several months before the nova outburst. A search through archival X-ray data showed the presence of an X-ray source, before the nova outburst, at the nova position (Ibarra \& Kuulkers 2008; Ibarra et al. 2008). Here we describe in more detail the serendipitous X-ray observations performed by ROSAT, XMM-Newton and Swift of the pre-nova. Preliminary results were reported by Ibarra \& Kuulkers (2008) and Ibarra et al. (2008). Since the discovery, follow-up X-ray observations have been made with Swift (Kuulkers et al. 2008; Page et al. 2008; Osborne et al. 2008; Page et al., in prep.), XMM-Newton (Ness et al. 2008a, 2008b, in prep.) and Suzaku (Takei et al., in prep.).

\section{X-ray observations}

A search of the X-ray catalogues (available through Vizier at the Centre de Données de Strasbourg) indicated that the field of V2491 Cyg was observed at different epochs before the nova outburst by ROSAT, XMM-Newton, and Swift. We determine the Swift position by combining all data into one single image and using the ximage tool. In Table 1, we list the positions and position errors of the candidate X-ray counterparts. In Fig. 1, we show a Swift/XRT image with all the pre-nova X-ray counterpart uncertainties. The following paragraphs describe these satellites and the relevant instruments. The results are summarised in Table 2.

The ROSAT satellite (Trümper et al. 1991) produced an all-sky survey (RASS) in the energy range $0.2-2.4 \mathrm{keV}$ that covered about $25 \%$ of the sky during pointing observations with the PSPC camera (Voges et al. 2000). Two X-ray sources in these catalogs have coordinates close to that of V2491 Cyg, i.e., the RASS source 1RXS J194259.9+321940 and the source 2RXP J194302.0+321912 (see also Table 1 and

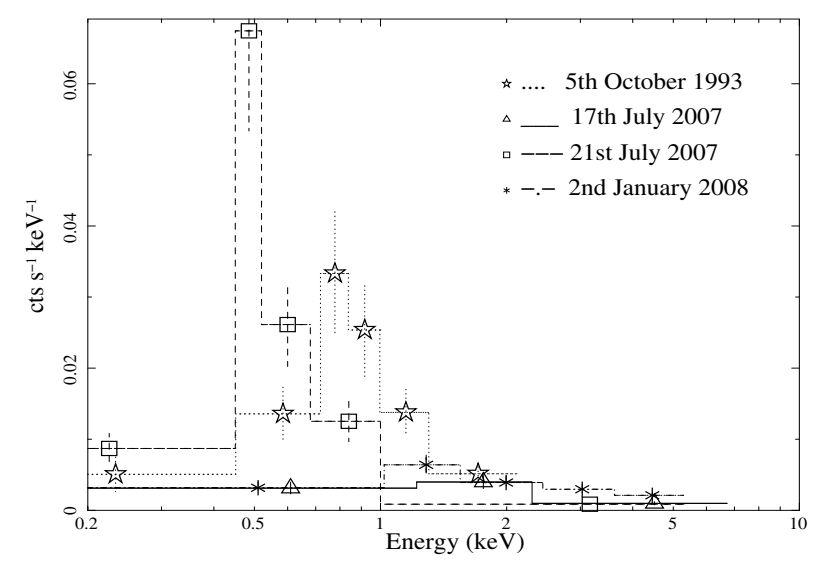

Fig. 2. ROSAT/PSPC (one) and Swift/XRT (three) spectra of pre-nova $\mathrm{X}$-ray observations. Shown are the data points for all the observations.

Fig. 1). We re-analyzed the 2RXP J194302.0+321912 observation using the HEASOFT software suite. We filtered the ROSAT event list with XSELECT, using an $80^{\prime \prime}$ source extraction region and a local background region.

XMM-Newton (Jansen et al. 2001) is performing a sensitive survey of the sky in the $0.2-12 \mathrm{keV}$ energy band, as it slews between targets. A catalogue of slew point sources was published by Saxton et al. (2008).

We report on XMM-Newton observations by the EPIC-pn camera (Strüder et al. 2001) operating in full-frame mode with the medium filter during a slew performed on 2006 November 15 (OBSID. 9127000003). V2491 Cyg passed through the EPIC-pn field of view at a large off-axis angle such that an effective onaxis exposure time of $3.4 \mathrm{~s}$ was achieved. A source with 4 photons was detected in the full $(0.2-12 \mathrm{keV})$ energy band of the EPIC-pn with a significance of $3.5 \sigma$ above the low background level $\left(0.2 \mathrm{cts} / \mathrm{arcmin}^{2}\right)$ of the slew image. No XMM-Newton pointed observations of this field were acquired before the outburst occurred.

Six Swift observations of the V2491 Cyg field were completed before the nova outburst, as part of a follow-up survey aimed at identifying the X-ray counterpart of sources discovered by the Burst Alert Telescope (BAT; Barthelmy et al. 2005). The survey (Tueller et al. 2008; Tueller et al., in prep.) targered Swift J1942.8+3220 (Sect. 3.3).

The Swift X-ray Telescope (XRT, 0.3-10 keV; Burrows et al. 2005) detector was operated in photon-counting (PC) mode, which provides two-dimensional imaging, spectral information, and a $2.5 \mathrm{~s}$ time resolution. All the Swift/XRT data were processed by XRTPIPELINE (Swift_Rel2.8), with local-background subtracted spectra binned to $\geq 20 \mathrm{cts} \mathrm{bin}^{-1}$. One of the XRT observations was too short (20 s on 2007 July 15) to be useful, and was not been included in our spectral analysis.

No optical Swift/UVOT data were available. This is because the UVOT telescope was operated with a BLOCKED filter due to the presence of a bright star in the field of view. 
Table 2. Observation log of the ROSAT, XMM-Newton and Swift pre-nova observations. Apart from the exposure time the count rates in different energy bands as well as the absorbed fluxes are given (see text for more details).

\begin{tabular}{|c|c|c|c|c|c|c|c|}
\hline Date & $\begin{array}{c}\text { Days } \\
\text { before } \\
\text { outburst }\end{array}$ & Instrument & $\begin{array}{c}\text { Exposure time } \\
(\mathrm{ks})\end{array}$ & $\begin{array}{l}\text { Count rate }^{1} \\
\text { (cts/ks) }\end{array}$ & $\begin{array}{c}\text { Count rate } \\
\text { (cts/ks) } \\
0.3-1.0 \mathrm{keV} \\
\end{array}$ & $\begin{array}{c}\text { Count rate } \\
\text { (cts } / \mathrm{ks}) \\
1.0-8.0 \mathrm{keV} \\
\end{array}$ & $\begin{array}{c}0.2-10.0 \mathrm{keV}^{-2} \mathrm{fl}^{2} \\
\left(10^{-12} \mathrm{erg} \mathrm{cm}^{-2} \mathrm{~s}^{-1}\right) \\
\text { absorbed }\end{array}$ \\
\hline 1990-10-19 & 6383 & ROSAT/PSPC[1RXS] & 0.39 & $28 \pm 11$ & - & - & $4.5_{-1.8}^{+19.5}$ \\
\hline 1993-10-05 & 5302 & $R O S A T / \mathrm{PSPC}[2 \mathrm{RXP}]$ & 4.3 & $22 \pm 3$ & $12 \pm 2$ & $8 \pm 2$ & $0.3 \pm 0.1$ \\
\hline 2006-11-15 & 517 & XMM-Newton/EPIC & $3.43 \times 10^{-3}$ & $1173_{-587}^{+613}$ & - & - & $2.5_{-1.6}^{+7.0}$ \\
\hline $2007-05-25$ & 322 & Swift/XRT & 1.06 & $21 \pm 5$ & $1.9_{-1.3}^{+1.9}$ & $20_{-4}^{+5}$ & $5.5_{-1.4}^{-1.6}$ \\
\hline $2007-06-10$ & 306 & Swift/XRT & 0.97 & $41 \pm 7$ & $11.8_{-36}^{-1.3}$ & $31_{-6}^{+7}$ & $10.7_{-2.2}^{+16.4}$ \\
\hline 2007-07-17 & 269 & Swift/XRT & 3.82 & $23 \pm 3$ & $3.5_{-1.1}^{+1.3^{\circ}}$ & $19 \pm 3$ & $1.0 \pm 0.2$ \\
\hline 2007-07-21 & 265 & Swift/XRT & 4.87 & $37_{-4}^{+3}$ & $27.9 \pm 3.2$ & $9 \pm 2$ & $0.8 \pm 0.2$ \\
\hline 2008-01-02 & 100 & Swift/XRT & 5.65 & $24 \pm 2$ & $3.9 \pm 1.0$ & $20 \pm 2$ & $1.6_{-0.4}^{+0.5}$ \\
\hline
\end{tabular}

Note 1: energy bands: ROSAT/PSPC, 0.2-2.0 keV; XMM-Newton/EPIC, 0.2-12 keV; Swift/XRT, 0.3-10 keV.

Note 2: for the second ROSAT observation, the XMM-Newton and the first two Swift observations, the flux was calculated using a power-law with $\Gamma$ between 1.3 and 5.2, and $N_{\mathrm{H}}=2.2 \times 10^{21} \mathrm{~cm}^{-2}$.

\section{Results}

\subsection{The pre-nova X-ray identification}

In Table 1, we give the positions and source names of the $\mathrm{X}$-ray sources that we identify close to the optical position of V2491 Cyg. Based on the Swift/XRT observations, the prenova X-ray source was designated as Swift J194302.1+321913 (Ibarra et al. 2008). The position of the ROSAT/PSPC source, 2RXP J194302.0+321912, the XMM-Newton/EPIC-pn source XMMSL1 J194301.9+321911, and the Swift/XRT source Swift J194302.1+321913 are all consistent with the optical nova coordinates (see also Ibarra \& Kuulkers 2008; Ibarra et al. 2008). The ROSAT/PSPC source position of 1RXS J194259.9+321940 is formally inconsistent with the optical position (see Fig. 1), although due to its large uncertainty, we consider that this is compatible with that of 2RXP J194302.0+321912. Based on the positional information, as well as the similar strength of the sources over time (see next subsection), we suggest that all the aforementioned X-ray sources are all the same source, and identify this to be the X-ray counterpart to the pre-nova of V2491 Cyg.

\subsection{Pre-nova $X$-ray spectral characteristics}

In Table 2, we list all X-ray pre-nova observations. For Swift/XRT, we provide the background corrected count rates in two bands, and the $68 \%$ confidence Bayesian rate errors (Kraft et al. 1991) because of the low numbers of counts. The source is clearly variable. Based on the information in the two energy bands, it's spectrum changes dramatically in shape between observations on a time scale down to 4 days. The softness of the source on 2007 July 21 is particularly noticiable (see also Fig. 1).

The second ROSAT and last three Swift observations provide sufficient counts for a more detailed X-ray spectral analysis. We first used a power-law model to describe our data and fit them with XSPEC version 11.3. Using this model and allowing $N_{\mathrm{H}}$ to be a free parameter in the fits led to poorly constrained values, especially for $N_{\mathrm{H}}$. To constrain $N_{\mathrm{H}}$ more reliably, we completed a simultaneous fit to the four individual spectra, using the same value of $N_{\mathrm{H}}$, while leaving the power-law parameters independent. This resulted in a reasonable fit $\left(\chi_{\text {red }}^{2}=\right.$ 1.2 with 10 d.o.f) with $N_{\mathrm{H}}=2.2_{-1.1}^{+1.3} \times 10^{21} \mathrm{~cm}^{-2}(90 \%$ statistical errors are quoted for all parameters). The photon index values are: $3.6_{-0.8}^{+0.7}$ for the ROSAT observation and $1.7_{-0.4}^{+0.5}$, $5.2_{-0.7}^{+1.2}$ and $1.2 \pm 0.4$ for the last three Swift observations, respectively. Our value of $N_{\mathrm{H}}$ is compatible with that derived from the optical measurements (see Sect. 1). The softest spectrum has a steep power-law index, compared to the other spectra. Although spectra of novae are known to show many emission lines (e.g., Mukai \& Orio 2005), X-ray spectral fits using other models (e.g., the optically-thin thermal plasma model VAPEC, with abundances of $\mathrm{C}$ and $\mathrm{Fe}$ fixed at 0.3 solar, and $\mathrm{N}$ fixed at 8 times solar, which are typical for novae, Nussbaumer et al. 1988; Ness et al. 2005, or Bremsstrahlung) did not provide improved fits. We also stacked all Swift observations (excluding the one taken 265 days before outburst; see Table 2) to get better statistics. Again, fits to the resulting spectrum provided a more accurate reproduction of the data when using a power law compared to a VAPEC model. Unfortunately, the limited quality of the data prevented us from investigating in more detail multitemperature emission-line models.

Using the best-fit power-law spectral fits, we derived unabsorbed X-ray $(0.2-10 \mathrm{keV})$ flux values of between 1 and $30 \times$ $10^{-12} \mathrm{erg} \mathrm{cm}^{-2} \mathrm{~s}^{-1}$, taking into account the observations from the various instruments.

\subsection{The unidentified source Swift J1942.8+3220}

It is worth considering whether V2491 Cyg might be the counterpart to the Swift/BAT detection Swift J1942.8+3220, whose error circle encompasses the nova position (see Fig. 1). The Swift/BAT survey catalog source Swift J1942.8+3220 has an estimated average flux of about $2 \times 10^{-11} \mathrm{erg} \mathrm{cm}^{-2} \mathrm{~s}^{-1}(14-195 \mathrm{keV}$; Tueller et al. 2008). Using public archival INTEGRAL/IBIS/ISGRI observations performed between March 2003 and December 2006 (for a total exposure time of about $620 \mathrm{ks}$ ), we derived a $3 \sigma$ upper limit $(18-50 \mathrm{keV})$ of about $1 \times 10^{-11} \mathrm{erg} \mathrm{cm}^{-2} \mathrm{~s}^{-1}$ at the position of V2491 Cyg, which is more or less comparable with that derived for the Swift/BAT source.

Apart from SwiftJ194302.1+321913, there is another XRT source within the $\sim 5^{\prime}$ BAT error circle of Swift J1942.8+3220 (see upper part of Fig. 1) called Swift J194245.9+322411 $(\mathrm{RA}=19 \mathrm{~h} 42 \mathrm{~m} 45.9 \mathrm{~s}$, Dec = $+32 \mathrm{~d} 24^{\prime} 10.7^{\prime \prime}$, with $90 \%$ confidence error radius of $\left.3.6^{\prime \prime}\right)$. This source has an absorbed AGN-like X-ray spectrum; a power-law fit gives $\Gamma=1.5_{-0.4}^{+0.3}$ and $N_{\mathrm{H}}=(2 \pm 1) \times 10^{21} \mathrm{~cm}^{-2}$ with an unabsorbed $0.3-10 \mathrm{keV}$ flux of $1.1 \times 10^{-12} \mathrm{erg} \mathrm{cm}^{-2} \mathrm{~s}^{-1}$. Our aforementioned ROSAT and XMM-Newton identifications are not compatible with this AGN-like XRT source. The position of this source was covered by the XMM-Newton Slew Survey, 
but no source was detected with a $2 \sigma$ upper limit of $2 \times$ $10^{-12} \mathrm{erg} \mathrm{cm}^{-2} \mathrm{~s}^{-1}(0.2-12 \mathrm{keV})$, assuming the AGN-like XRT spectrum. The AGN-like XRT source is not bright enough to account for the BAT detection, assuming it is constant, and so it is unlikely to be the X-ray counterpart to the BAT source. On the other hand, if we extrapolate the power-law fits of the Swift/XRT spectra of the prenova V2491Cyg taken on 2007 July 17 and 2008 Jan. 2 (i.e., when the source was hard) into the BAT band, we infer fluxes that are more or less consistent with the BAT measured flux. If, however, the spectrum is assumed to be due to optically thin high-temperature thermal emission, the extrapolated flux is a few orders of magnitude lower. It remains, therefore, uncertain whether the BAT source is related to the pre-nova.

\section{Discussion}

We have identified a persistent, but variable, and, at least on one occasion, soft X-ray source present at the position of the nova V2491 Cyg from the ROSAT survey era up to 3 months before the nova outburst. This is only the second nova to be detected in X-rays before eruption (after V2487 Oph, Hernanz \& Sala 2002). A hard spectral component is suggested by Swift/BAT, but the association with the pre-nova is insecure.

V2487 Oph, also a fast nova $\left(t_{2} \simeq 6.3\right.$ days, see Hernanz \& Sala 2002), was proposed to be a recurrent nova, because of both the rapid decline in the optical and the presence of a plateau phase during the decline (Hachisu et al. 2002). Pagnotta et al. (2008) discovered a previous outburst of V2487 Oph in 1900, confirming its recurrent nature. V2491 Cyg was also suggested to be a recurrent nova (Tomov et al. 2008b).

Assuming a distance of $10.5 \mathrm{kpc}$ (Helton et al. 2008) and using the power-law fit results, we derived $0.2-10 \mathrm{keV} X$-ray luminosities ranging from about $1 \times 10^{34}$ to $4 \times 10^{35} \mathrm{erg} \mathrm{s}^{-1}$. This is comparable to that derived for V2487 Oph, about 3 years after the outburst, i.e., $\simeq 8 \times 10^{34} \mathrm{erg} \mathrm{s}^{-1}(0.2-10 \mathrm{keV}$; Hernanz et al. 2008) assuming a distance of $10 \mathrm{kpc}$ (but we note that the distance is rather uncertain; see Hernanz \& Sala 2002). It is, however, orders of magnitude higher than that seen for the recurrent nova RS Oph about 2 years after the 2006 outburst $(\simeq 5 \times$ $10^{31} \mathrm{erg} \mathrm{s}^{-1}$, Nelson \& Orio 2008, using a distance of $1.6 \mathrm{kpc}$; Mason et al. 1987; Bode et al. 2008). This is compatible with the fact that in general fast novae appear to be brighter than slow novae, when they are at quiescence (e.g., Becker \& Marshall 1981; Orio et al. 2001).

Our X-ray luminosity estimates imply inter-nova mass accretion rates in the range $10^{-9}-10^{-8} M_{\odot} \mathrm{yr}^{-1}$, for a $1 M_{\odot}$ white dwarf. These rates are around an order of magnitude lower than those required (judged from the models of Yaron et al. 2005) to fuel the novae outbursts of RS Oph, which are recurrent on a 20 year period. This would imply that, even with a massive white dwarf, the recurrence timescale for novae in V2491 Cyg would be $>\sim 100 \mathrm{yrs}$, similar to that inferred for V2487 Oph.

The soft spectra observed with ROSAT/PSPC and Swift/XRT resemble those of the post-outburst super-soft state (Page et al. 2009 , in prep.), but are much fainter. This, and the fact that the Swift/XRT observation was taken less than a year before the nova outburst, suggests that the spectra were not obtained during a super-soft state after a previous nova outburst or accretiondriven nuclear burning, in agreement with our relatively low mass-transfer estimates. The Swift/XRT observations show that V2491 Cyg changes spectral state on at least a 4-day timescale.

Magnetic CVs in quiescence show harder spectra than their non-magnetic equivalents (e.g., Barlow et al. 2008;
Landi et al. 2009). If the BAT source is the same as that seen at $\lesssim 10 \mathrm{keV}$, V2491 Cyg may be magnetic. Polars, for example, are known to change from hard to soft states as the white dwarf rotates (e.g., Heise et al. 1985), which could explain the X-ray spectral evolution that we observe. However, we see no evidence for short-term ( hour) orbital related variations. In general, polars are rather weak X-ray sources (see King \& Watson 1987). Additionally, at $\lesssim 10 \mathrm{keV}$, the spectrum is not unlike that seen in non-magnetic CVs (e.g., Baskill et al. 2005). This brings into doubt a magnetic interpretation.

Acknowledgements. We acknowledge the use of observations obtained with $X M M-N e w t o n$, an ESA science mission with instrument and contributions directly funded by ESA Member States and the USA (NASA), as well as public data from the Swift data archive. J.O. and K.P. acknowledge the support of the STFC; S.S. acknowledges partial support from NASA and NSF grants to ASU.

\section{References}

Ashok, D. P., Barnerjee, D. P. K., Joshi, V., \& Naik, S. 2008, CBET, 1354 Ayani, K., \& Matsumoto, K. 2008, CBET, 1334

Balman, S., Pekon, Y., \& Kiziloglu, U. 2008, ATel, \#1504

Barlow, E. J., Knigge, C., Bird, A. J., et al. 2006, MNRAS, 372, 224

Barthelmy, S. D., Barbier, L. M., Cummings, J. R., et al. 2005, SSRv, 120, 143

Baskill, D. S., Wheatley, P. J., \& Osborne, J. P. 2005, MNRAS, 357, 626

Becker, R. H., \& Marshall, F. E. 1981, ApJ, 244, L93

Bode, M. F., \& Evans, A. 2008, in Classical Novae, CUP

Burrows, D. N., Hill, J. E., Nousek, J. A., et al. 2005, SSRv, 120, 165

Hachisu, I. 2002, ASP Conf. Ser., 261, 629

Heise, J., Brinkman, A. C., Gronenschild, E., et al. 1985, A\&A, 148, L14

Helton, L. A., \& Woodward, C. E. 2008, CBET, 1379

Hernanz, M. 2008, in The X-ray Universe Symposium, http://xmm.esac. esa.int/external/xmmscience/workshops/2008symposium/ Hernanz, M., \& Sala, G. 2002, Science, 298, 393

Ibarra, A., \& Kuulkers, E. 2008, ATel, \#1473

Ibarra, A., Kuulkers, E., Beardmore, A., et al. 2008, ATel, \#1478

Jansen, F., Lumb, D., Altieri, B., et al. 2001, A\&A, 365, L1

Jurdana-Sepic, R., \& Munari, U. 2008, IBVS, 5839

King, A. R., \& Watson, M. G. 1987, MNRAS, 227, 205

Kraft, R. P., Burrows, D. N., \& Nousek, J. A. 1991, ApJ, 374, 344

Landi, R., Bassani, L., Dean, A. J., et al. 2009, MNRAS, 392, 630

Lynch, D. K., Russell, R. W., \& Rudy, R. J. 2008, IAU Circ., 8935

Kuulkers, E., Ibarra, A., Page, K. L., et al. 2008, ATel, \#1480

Mason, K. O., Córdova, F. A., Bode, M. F., \& Barr, P. 1987, in RS Oph (1985) and the Recurrent Nova Phenomenon, ed. M. F. Bode (Utrecht: VNU Science Press), 167

Mukai, K., \& Orio, M. 2005, ApJ, 622, 602

Nakano, S., Beize, J., Jin, Z.-W., et al. 2008, IAU Circ., 8934

Nelson, T., \& Orio, M. 2008, ATel, \#1494

Ness, J.-U., Starrfield, S., Jordan, C., Krautter, J., \& Schmitt, J. H. M. M. 2005, MNRAS, 364, 1015

Ness, J.-U., Starrfield, S., Gonzalez, R., et al. 2008a, ATel, \#1561

Ness, J.-U., Starrfield, S., Gonzalez, R., et al. 2008b, ATel, \#1573

Nussbaumer, H., Schmid, H. M., Vogel, M., \& Schild, H. 1988, A\&A, 198, 179

O’Brien, T. J., Lloyd, H. M., \& Bode, M. F. 1994, MNRAS, 271, 155

Orio, M., Covington, J., \& Ögelman, H. 2001, A\&A, 373, 542

Osborne, J. P., Page, K., Evans, P., et al. 2008, ATel, \#1542

Page, K. L., Osborne, J. P., Evans, P. A., et al. 2008, ATel, \#1523

Pagnotta, A., Schaefer, B. E., \& Xiao, L. 2008, IAU Circ., 8951

Predehl, P., \& Schmitt, J. H. M. M. 1995, A\&A, 293, 889

Rudy, R. J., Lynch, D. K. \& Russell, R. W. 2008, IAU Circ., 8938

Saxton, R. D., Read, A. M., Esquej, P., et al. 2008, A\&A, 480, 611

Strüder, L., Briel, U., Dennerl, K., et al. 2001, A\&A, 365, 18

Tomov, T., Mikolajewski, M., Ragan, E., Swierczynski, E., \& Wychudzki, P. 2008a, ATel, \#1475

Tomov, T., Mikolajewski, M., Brozek, T., et al. 2008b, ATel, \#1485

Trümper, J., Hasinger, G., Aschenbach, B., Braeuninger, H., \& Briel, U. G. 1991, Nature, 349, 579

Tueller, J., Mushotzky, R. F., Barthelmy, S., et al. 2008, ApJ, 681, 113

Voges, W., Aschenbach, B., Boller, T., et al. 2000, IAUC, 7432

Williams, R. E. 1992, ApJ, 104, 725

Yaron, O., Prialnik, D., Shara, M. M., \& Kovetz, A. 2005, ApJ, 623, 398 